\title{
A Review on Olefin Metathesis Reactions as a Green Method for the Synthesis of Organic Compounds
}

\author{
Atitegeb Abera Tsedalu \\ Department of Chemistry, Arba Minch University, Arba Minch, Ethiopia \\ Correspondence should be addressed to Atitegeb Abera Tsedalu; atitegeb.abera@amu.edu.et
}

Received 15 August 2020; Revised 4 February 2021; Accepted 9 July 2021; Published 6 September 2021

Academic Editor: Pasquale Longo

Copyright (C 2021 Atitegeb Abera Tsedalu. This is an open access article distributed under the Creative Commons Attribution License, which permits unrestricted use, distribution, and reproduction in any medium, provided the original work is properly cited.

Olefin metathesis is a metal-mediated C-C bond exchange by which the two fragments within the olefin precursor are redistributed as a result of breaking the double bond to obtain a new product. Currently, most of the synthetic organic compounds, polymers, drugs, plastics, and other synthetic materials are synthesized via the application of olefin metathesis reactions. In this review, different types of olefin metathesis reactions with their plausible mechanisms and their application in synthetic organic chemistry have been discussed.

\section{Introduction}

There are many chemical transformation ways that are nonmetal processes that employ reactive functional groups, like aldehydes and ketones, to form carbon-carbon bonds for the synthesis of natural and synthetic organic compounds applicable for different purposes. Once these reactive functionalities are introduced, the subsequent cross-coupling reactions are very reliable double bond-forming processes. In many cases, however, protective groups are required to mask these functional groups prior to their conversion to olefins, such as carbonyl protective groups. Another drawback of these traditional methods is the use of harsh reagents, such as triflic anhydride and brominating reagents to prepare cross-coupling reagents. The most obvious reactions for $\mathrm{C}-\mathrm{C}$ bond formation which are the Wittig and Grignard reactions require highly basic conditions that are incompatible with many substrates of interest. In the case of Wittig reactions, every mole of the desired carbon-carbon double bond comes along with an equivalent of triphenylphosphine oxide, which is difficult to remove on a large scale as a waste of raw materials [1-3].

The following reactions (Schemes 1(a) and 1(b)) are examples of old methods to form $\mathrm{C}-\mathrm{C}$ double bonds using different reaction routes.
The aforementioned old methods have been proved in a synthesis framework of organic chemistry for C-C double bond formation with their drawbacks. But because of the above overwhelming problems, the effective, environmentally friendly, and elegant method which is the olefin metathesis reaction has been viewed as a synthetic route for the synthesis of target organic compounds. This reaction consists of a metal-catalyzed carbon skeleton redistribution in which a mutual exchange of unsaturated carbon-carbon bonds takes place, as illustrated in the catalyzed self-reaction of propene leading to ethene and but-2-ene shown in Scheme $2[4,5]$.

\section{Olefin Metathesis}

Olefin metathesis is a metal-catalyzed transformation, which acts on carbon-carbon double bonds and rearranges them via cleavage and reassembly [6-10]. This type of reaction is relatively simple and it often creates fewer undesired byproducts and hazardous wastes than alternative organic reactions. Most of the olefin metathesis reactions are transition metal alkylidene-catalysed reactions. These constitute a facile and efficient strategy for approching alkene precursors for the synthesis of various synthetic and natural products through olefin metathesis reactions. Such reaction 


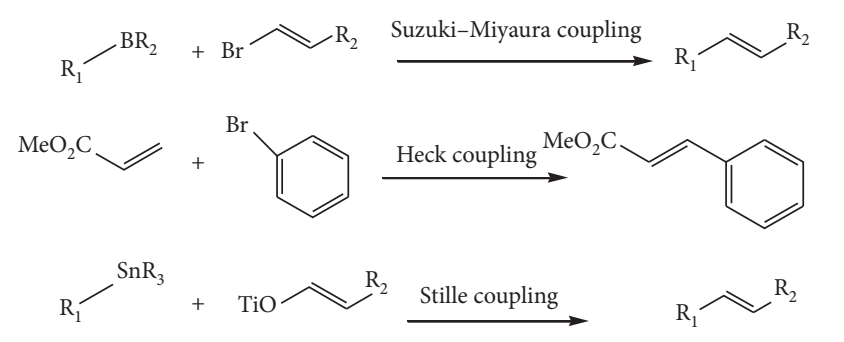

(a)

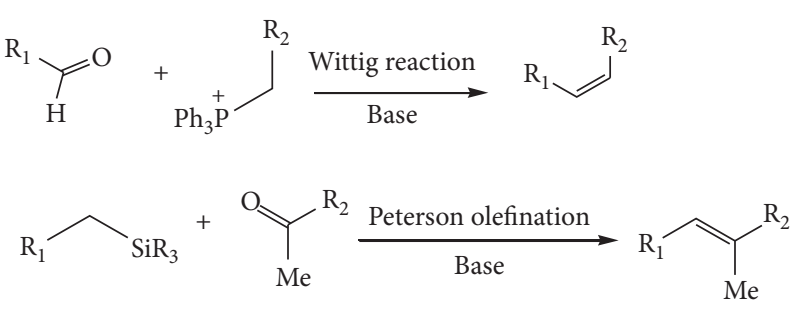

(b)

Scheme 1: (a) Metal-catalyzed alkene formation reactions. (b) Alkene formation reactions without metal catalyst.

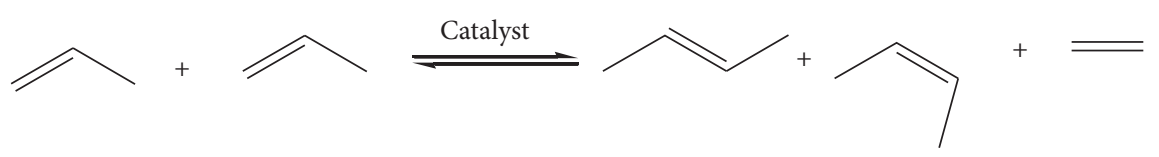

Scheme 2: Metathesis reaction of 1-propene to form trans and cis-2-butene.

routes have been emerged in due time in different effortful research studies in different industries and academia $[11,12]$.

Olefin metathesis is one of the very few fundamentally novel organic reactions which open up new industrial routes to important petrochemicals, polymers, oleochemicals, and specialty chemicals applicable for different purposes [13]. This type of reaction is now being used in drug discovery for the synthesis of anticancer and other antibiotic organic compounds. Mazur et al. discovered a novel drug, the ansaferrocene-triazole-uracil conjugate, $( \pm)-9$ for the relief of breast cancer through metathesis reactions using $1,1^{\prime}$-diallylferrocene (1) as a starting material through ring-closing metathesis and other consecutive reactions in the presence of Grubbs catalysts (Schemes 3(a)-3(c)) [14].

Development of metathesis catalysts (Figure 1) and improving different metathesis reactions for the synthesis of useful polymers and novel materials have been of interest to many researchers. In fact, the availability of various metathesis catalysts together with research efforts focused on the development of more active complexes helps to improve the economical outcome of industrial processes including those in the pharmaceutical industry [15].

On the other hand, a metal alkylidene catalyst-free metathesis reaction for the construction of the $\mathrm{C}-\mathrm{C}$ double bond is now taking view and interest in many organic laboratories. Haung et al. (2016) synthesized tetrasubstituted quinolinones using diazo compounds and para-quinone methides via metathesis reactions using $\mathrm{TiCl} 4$ as the Lewis acid and dichloromethane as a solvent (Scheme 4) [16].

2.1. General Reaction Mechanism of Transition Metal Alkylidene Olefin Metathesis. Though the olefin metathesis reaction was discovered in the mid-1950s, its accepted mechanism (Scheme 5) was proposed by Chauvin and Herisson in 1971 which indicated that the reaction is catalyzed by metal carbenes [12].
According to this mechanism (Scheme 5), the coordination of an olefin to a metal-carbene catalytic species leads to the reversible formation of a metallacyclobutane intermediate. The metallacyclobutane can eliminate an olefin from either side of the ring, leading to degenerate metathesis, in which the starting olefin and carbene are reformed (nonproductive path), or productive metathesis, in which new olefins and carbenes are produced. As the catalytic cycle continues, an equilibrium mixture of olefins is produced, and the ultimate product ratio is determined by thermodynamic parameters. For instance, if one of the olefins is volatile, it can be removed from the system to drive the equilibrium towards the desired products [12].

There are two major approaches that are commonly employed to drive the reaction towards the desired products. One tactic is to rely on Le Chatelier's principle by continuously removing one of the products from the reaction system in order to shift the equilibrium in favor of the other product. This method is especially effective in the case of cross-metathesis (CM) reactions involving terminal olefins, ring-closing metathesis (RCM), and acyclic diene metathesis polymerization (ADMET) because the volatile gas byproduct (like ethene and propene) formed in these processes can be easily removed [17-25].

The other approach capitalizes on the ring strain of cyclic olefins such as cyclooctenes and norbornenes. The energy released during the ring-opening reaction of these compounds is sufficient to drive reactions such as ring-opening cross metathesis (ROCM) and ring-opening metathesis polymerization (ROMP) forward. In some instances, substrate concentration, which often distinguishes ADMET from RCM or the catalysts' sensitivity to olefin substitution, can also be taken advantage of to influence product selectivity. All of these methods are currently successfully employed in the synthesis of a large variety of small, medium, and polymeric molecules, as well as novel materials [6, 7, 24, 26-35].

Since all of these processes are fully reversible, only statistical mixtures of starting materials as well as all of the 


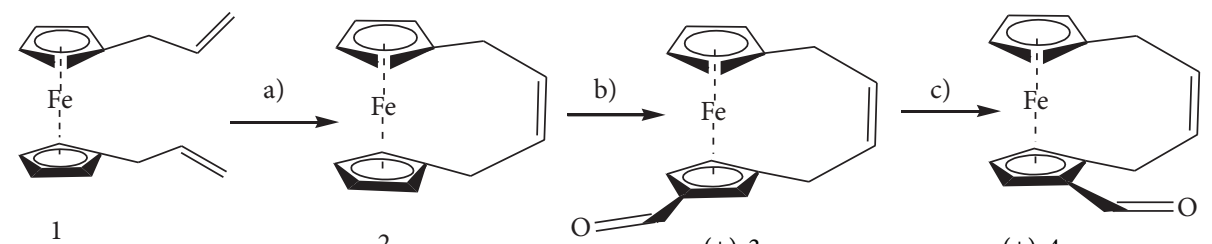

( \pm )-3

( \pm$)-4$

(a)

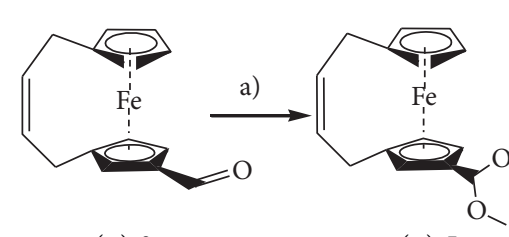

( \pm )-3

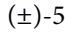

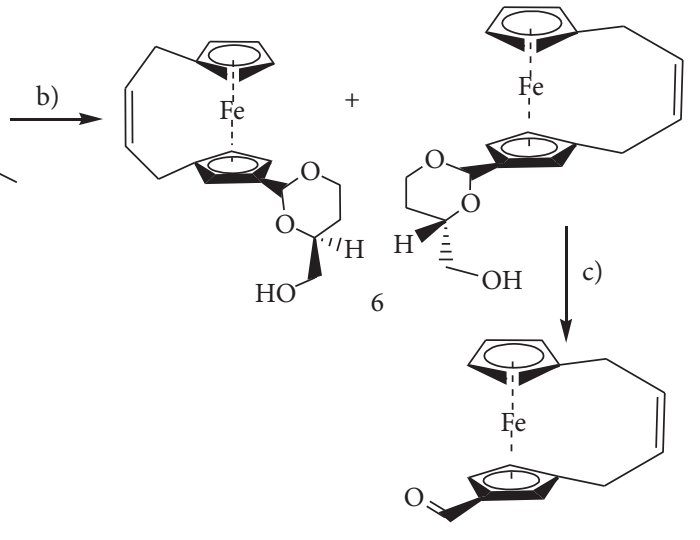

$( \pm)-3^{\prime}$

(b)

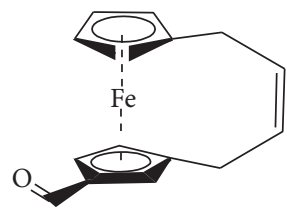

$( \pm)-3^{\prime}$

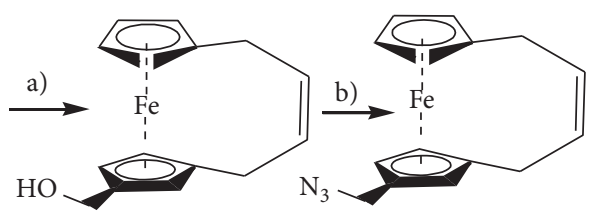

(土)-7

( \pm )-8

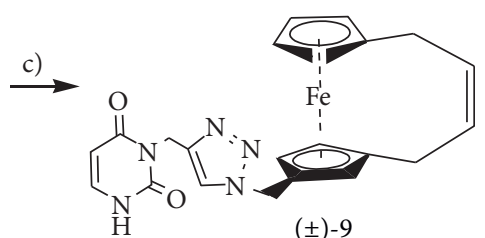

(c)

Scheme 3: (a) Synthesis of the ( \pm )-3and ( \pm )-4 aldehydes: a) $\left[\mathrm{Ru}(=\mathrm{CHPh}) \mathrm{Cl}_{2}\left(\mathrm{PCy}_{3}\right)_{2}\right], \mathrm{CH}_{2} \mathrm{Cl}_{2}$, reflux, $\left.3 \mathrm{~h} ; \mathrm{b}\right) \mathrm{CH}(\mathrm{OEt})_{3}, \mathrm{AlCl}{ }_{3}$, toluene, $0^{\circ} \mathrm{C}$ to rt, $1 \mathrm{~h}$ or DMF, $\mathrm{POCl}_{3}, \mathrm{CHCl}_{3}$, rt, $20 \mathrm{~h}$. (b) Resolution of ( \pm )-3 into enantiomers: a) $\mathrm{CH}(\mathrm{OMe})_{3}, \mathrm{p}-\mathrm{TsOH}, 80-90^{\circ} \mathrm{C}$; b) (S)-(-)-1,2,4butanetriol, p-TsOH, $\mathrm{CHCl}_{3}, 60^{\circ} \mathrm{C}$; then crystallization from hexanes and 2-propanol at $4^{\circ} \mathrm{C}$; c) p-TsOH, $\mathrm{CH}_{2} \mathrm{Cl}_{2}, \mathrm{H}_{2} \mathrm{O}, 60^{\circ} \mathrm{C}$. (c) Synthesis of ( \pm )-9: a) $\mathrm{NaBH}_{4}, \mathrm{MeOH}$, THF, rt, $24 \mathrm{~h}$ for $7 \mathrm{a} ; 48 \mathrm{~h}$ for ( \pm )-7; b) $\mathrm{NaN}_{3}, \mathrm{CH}_{3} \mathrm{COOH}, 50^{\circ} \mathrm{C}, 3 \mathrm{~h}$; c) 3-propargyluracil, $\mathrm{CuSO}_{4} \times 5 \mathrm{H}_{2} \mathrm{O}$, sodium ascorbate, EtOH, rt 4 days for $( \pm)-9$.

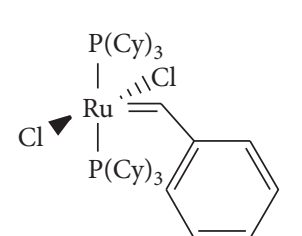

G-I

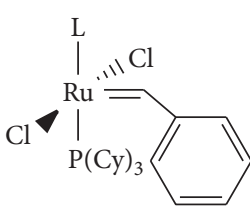

G- II; G- II'<smiles>CC(C)Oc1ccccc1Cl</smiles>

H- 4<smiles>CC(C)Oc1ccccc1Cl</smiles>

H-II; H-II'<smiles>CC(C)Oc1ccc([N+](=O)[O-])cc1Cl</smiles>

E-II; E-II'<smiles>COC(=O)C(C)Oc1ccccc1Cl</smiles>

E-II; E-II'<smiles>CCC[R1](Cl)(Cl)=C1C=C(c2ccccc2)c2ccccc21</smiles>

Ind-II; Ind-II'

Figure 1: Selected modern ruthenium-based olefin metathesis catalysts. 


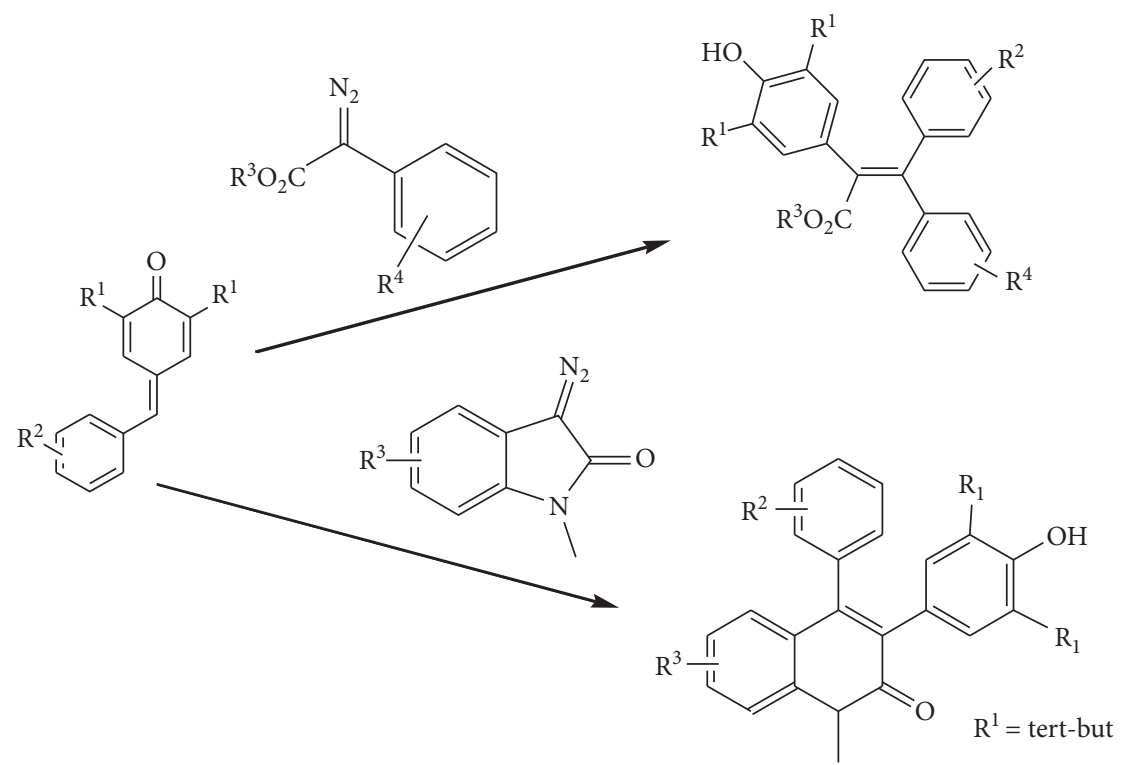

SCHEme 4: Synthesis of tetrasubstituted quinolinones using diazo compounds and para-quinone methides via metathesis reactions using ticl 4 as the Lewis acid and dichloromethane as a solvent.

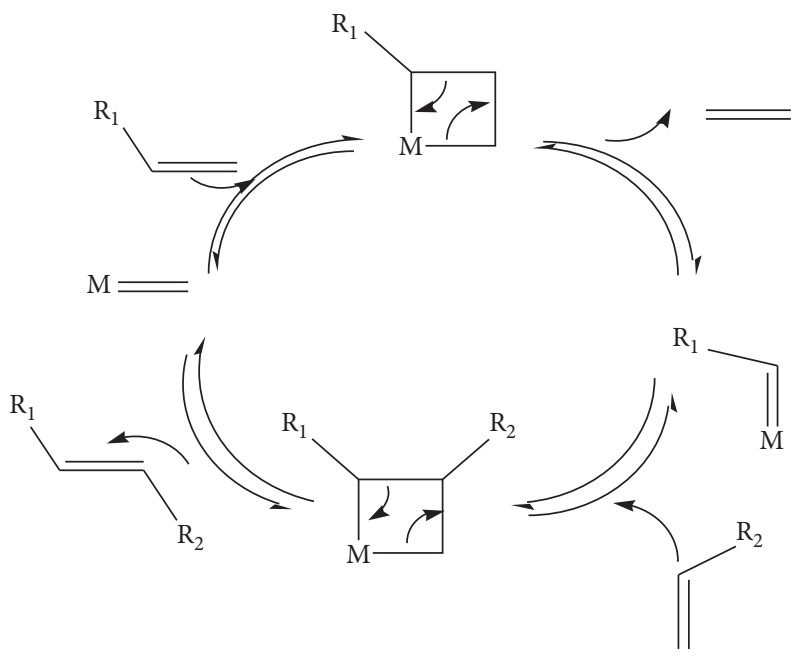

Scheme 5: General olefin metathesis reaction mechanism.

possible rearrangement products are produced in the absence of thermodynamic driving forces $[11,36]$.

During the olefin metathesis reactions, many side reactions which may appear like isomerization (especially double bond shift reactions), alkylation, cyclization, and addition across the double bond) are challenging problems. These problems are mostly created due to the residual ruthenium catalyst and can generally be avoided by proper choice of solvent as well as other purification techniques. For example, diethyl ether can leach ruthenium in a reaction with highly polar substrates like alcohols and acids. On the other hand, catalyst I can be removed by $\mathrm{SiO} 2$ and DMSO at room temperature for 12 hours $[37,38]$.

\section{Olefin Metathesis Reaction Types}

Olefin cross metathesis (CM) represents an alternative to the olefination methods where olefins themselves are the reactive functional groups (Scheme 6).

Investigations into olefin cross metathesis with several classes of olefins, including substituted and functionalized styrenes, secondary allylic alcohols, tertiary allylic alcohols, and olefins with alpha-quaternary centers, have led to a general model useful for the prediction of product selectivity and stereoselectivity in cross metathesis. As a general ranking of olefin reactivity in CM, olefins can be categorized (Scheme 7) into four by their relative abilities to undergo 


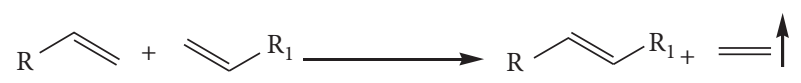

Scheme 6: General reaction for cross olefin metathesis.

homodimerization via cross metathesis and the susceptibility of their homodimers towards secondary metathesis reactions due to steric and electronic influence. By employing a metathesis catalyst with the appropriate activity as well as proper olefinic partners, selective cross-metathesis reactions can be achieved with a wide variety of electronrich, electron-deficient, and sterically bulky olefins $[39,40]$ :

Type I: rapid homodimerization, homodimers consumable (i.e., reversible reaction)

Type II: slow homodimerization, sparingly consumable (i.e., somewhat reversible reaction)

Type III: no homodimerization

Type IV: olefins inert to cross metathesis but do not interfere with catalyst activity towards other alkenes

When two type I olefins are paired in a CM reaction, the rates of homodimerization and cross-product formation are similar, and the reactivities of the homodimers and cross products towards secondary metathesis events can be high. In these reactions, the desired cross product will be formed at a rate comparable to the homodimers, and equilibration of the cross products with the various homodimers through secondary metathesis reactions will result in a statistical product mixture $[39,41]$.

Olefin category is done based on reactivity towards cross-metathesis reactions with Grubbs-II catalyst (the above figure). Therefore, the selective cross-metathesis reaction will occur based on the type of olefinic partner as well as the catalyst reactivity ( $\mathrm{N}$-heterocyclic carbene ligand containing catalysts such as the second-generation Grubbs catalyst 1 in Figure 2 are good) [15, 42-44].

Much amount of catalyst and heat should be required to carry out cross olefin metathesis reactions with some challenging olefinic partners such us vinyl ketones $[45,46]$ acrylic acid [47, 48], and acrylonitrile [49-52]. To make the reaction facile and fast for those substrates, Voigtritter et al. described a new procedure for carrying out CM reactions (Scheme 8) with Grubbs-II catalyst under the assistance of a copper(I) salt, CuI, which not only leads to faster rates of cross couplings but avoids chlorinated solvents as well [53].

As the study indicated that iodine may serve as a stabilizing ligand on ruthenium so that it extends the lifetime of the Grubbs catalyst. Therefore, CuI provides two positive effects. One is the ligand effect of iodide on ruthenium and the second is a phosphine sequestering effect by copper(I) from ruthenium. In this procedure, even very challenging type II and type III olefinic partners listed above (e.g., acrylic acid, acrylonitrile, and isopropylidene derivatives) were involved in cross metathesis (Schemes 8(b) and 8(c)).

Ring-Closing Metathesis, RCM. It is the intramolecular metathesis of a diene to form a cyclic olefin which is used for synthesizing both carbocyclic and heterocyclic compounds
[43]. Olefin metathesis, as shown by the mechanism proposed by Chauvin, is a process that is normally fully reversible. This implies that a driving force must be present in order to obtain a single, defined product. For RCM and CM, the equilibrium is usually driven in the desired direction by progressive removal of the by-product from the reaction environment (commonly ethene or propene, liberated as gas). Examples of RCM reactions in which nonvolatile products are produced (e.g., styrene) are known and for specific applications, important results have been obtained $[54,55]$.

RCM helps to transform linear substrates to cyclic olefins (the following scheme) and therefore is frequently used in the preparation of biologically important compounds containing medium- and large-size rings as well as capsules of varying sizes, properties, and applications. Indeed, RCM targets modification of macrostructure rather than the introduction of new functionality $[56,57]$.

This type of metathesis reaction has also realized the possibility of constructing carbocyclic rings of both small and large sizes with the potential of accessing a variety of heterocyclic systems (Scheme 9) [10, 58].

The most widely used catalysts for olefin ring-closing metathesis are Grubb's ruthenium alkylidene catalysts (Figure 2) which exhibit high reactivity in a variety of RCM processes and show remarkable tolerance towards many different organic functional groups.

\section{Mechanism of Ring-Closing Metathesis (RCM)}

RCM reactions like the other olefin metathesis reactions proceed via metallacyclobutane and metal-carbene intermediates (Scheme 10) which involve effectively a series of alternating [2+2] cycloadditions and cycloreversions between metal alkylidene and metallacyclobutane species. In the first turn of the catalytic cycle, the alkene byproduct depends on the $\mathrm{R}$ group in the original catalyst, while in the second and subsequent catalytic cycles, it depends on the substrate. For terminal alkene substrates, the reaction by-product is ethene. Alkene substitution in both substrate and catalyst can dramatically influence the reaction rate and outcome. In particular, several catalysts do not tolerate excessive steric hindrance on the substrate $[58,59]$.

Consider a typical RCM of hepta-1,6-diene shown as follows:

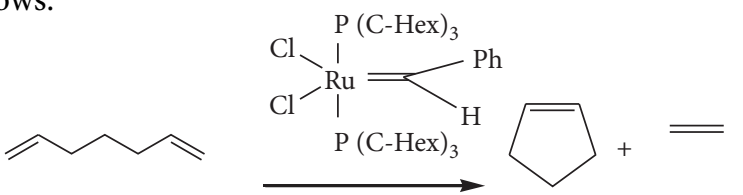

Ring-closing metathesis has been made for macrocarbocyclization and macrolactonization reactions 


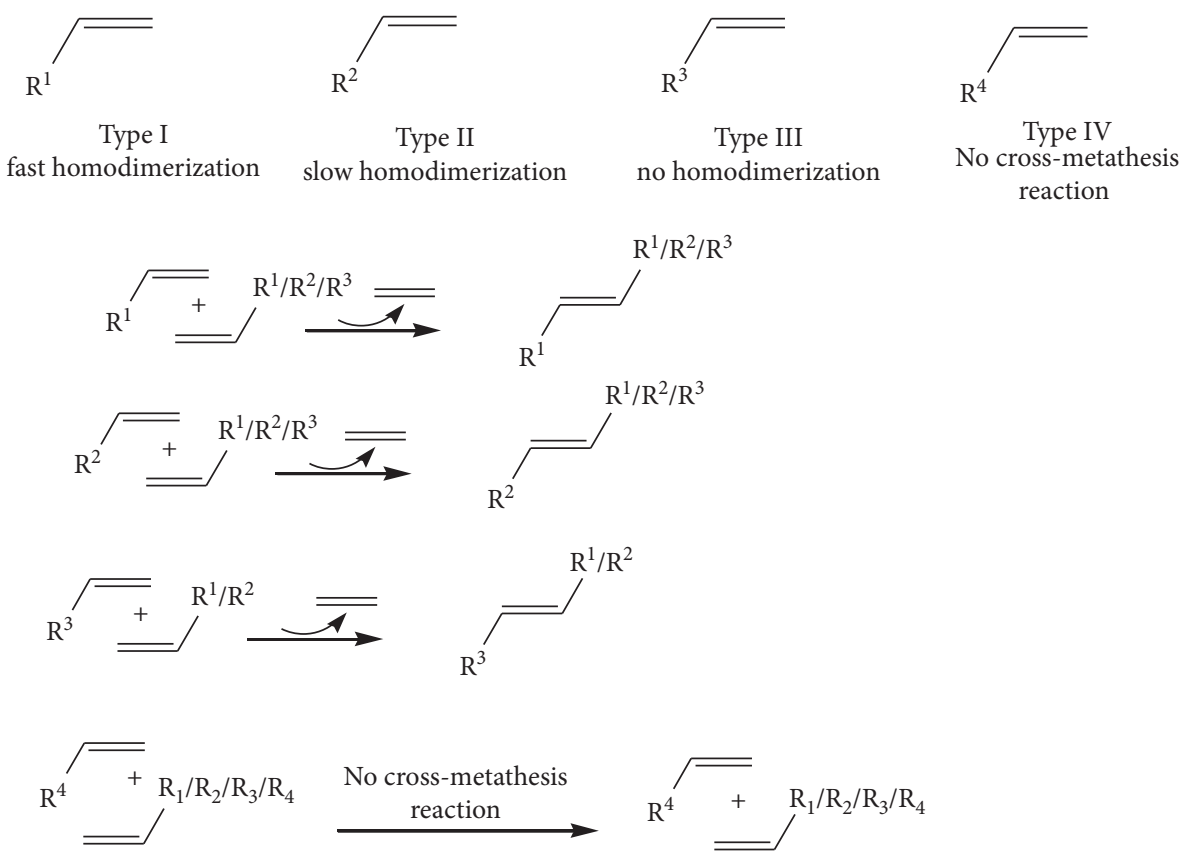

Scheme 7: Olefin categorization into type I, type II, type III, and type IV and their reactivity.

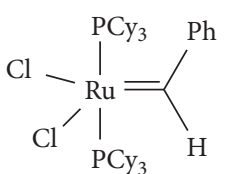

Grubbs 1st generation catalyst<smiles></smiles>

Grubbs-2 (1)

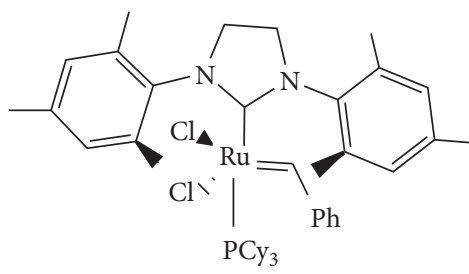

Grubbs- $2(2)$<smiles>CCCC(C)(Cl)[R1](Cl)(Cl)C=CC=C(c1ccccc1)c1ccccc1</smiles>

FIGURE 2: Structures of Ru-based catalysts used for olefin metathesis.

(formation of large ring cyclic esters) which had been difficult to take place now as a routine exercise in organic synthesis. Pharmaceutically active compounds having complex rings can be also produced via RCM reactions (Scheme 11) using Grubbs first generation catalyst [57].

RCM combined with CM has also been used in the total synthesis of bioactive natural products and producing functionalized oligomers, commonly known as telechelic materials. These materials are normally difficult to prepare in high yield and are finding growing application in the production of multiblock polymers such as styrene-butadienestyrene and thermoplastic polyurethanes $[40,57]$.

\section{Effect of Substituents on Ring-Closing Metathesis Reactions}

Electron-rich olefins make the ring-closing metathesis reaction fast (Figure 3(a)), whereas olefin precursors having electron-withdrawing groups retard the reaction. Indeed, if the heteroatom is present in the olefin precursor, it competes with the olefinic double bond to coordinate and react with the catalyst so as to be problematic to get the target product (Figure 3(b)) [61-63].

The success of RCM depends on to what extent the competing acyclic diene metathesis polymerization 


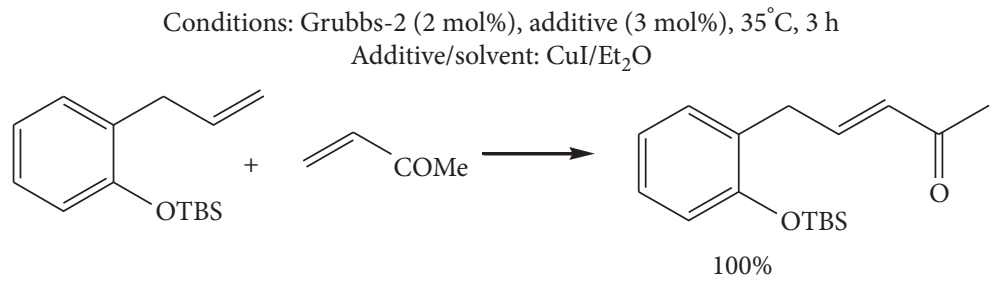

(a)<smiles>COc1ccc2ccc(=O)oc2c1CC=C(C)C</smiles><smiles>C=CC(=O)OC(C)(C)C</smiles><smiles>CCCCCCCCCCCOCC</smiles>
$\mathrm{Et}_{2} \mathrm{O}(0.1 \mathrm{M}), 35^{\circ} \mathrm{C}$

(b)<smiles>C=CCc1ccccc1OC</smiles>

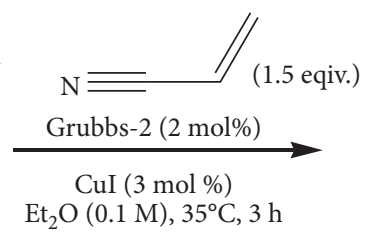

(c)<smiles>COc1ccc2ccc(=O)oc2c1CC=CC(=O)OC(C)(C)C</smiles>

$81 \%$<smiles>Cc1ccccc1CC=CC#N</smiles>

SCHEme 8: (a) Full conversion of the substrate into product with the effect of CuI. (b) Copper-assisted cross metathesis on osthole. (c) Effect of $\mathrm{CuI}$ on cross metathesis with acrylonitrile.

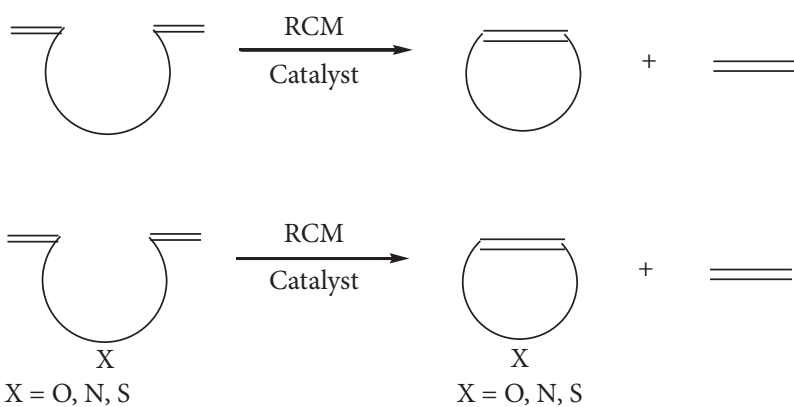

Scheme 9: General reaction of RCM for the synthesis of carbocyclic and heterocyclic molecules.

(ADMET) can be overcome. Decreasing the concentration of the substrate helps to reduce ADMET to some extent but this affects negatively the performance of the catalyst. In general, the ring-closure olefin metathesis reaction is influenced to a great extent by a number of factors. These include (1) the efficiency of the catalyst, (2) the nature of the resulting rings, (3) the size of the rings to be formed, and (4) the functional groups/substituents present in the substrate [59].

\section{Ring-Opening Metathesis Polymerization}

Ring-opening metathesis polymerization is a process of one or more cyclic olefins transformation to polymer catalyzed by metal-carbene compounds. Indeed, the number of double bonds both in polymer and in monomer is equal $[59,64]$.
The driving force behind the ROMP reaction (Scheme 12) of cyclic olefins is reliant on the release of ring strain energy, which compensates for the loss in entropy upon polymerization. Typically, ROMP is a thermodynamically favored process for cyclic olefins that have lower ring strain energy by which equilibrium will be formed between the monomers and polymers $[65,66]$.

To overcome this problem, the position of the equilibrium can be shifted to the ring-opened polymer via two approaches;

One involves lowering the temperature and the second involves increasing the monomer concentration. During ROMP (Scheme 13), the initiation step involves the coordination of a cyclic olefin to a metal alkylidene complex. Subsequent $[2+2] \quad$ cycloaddition produces a 


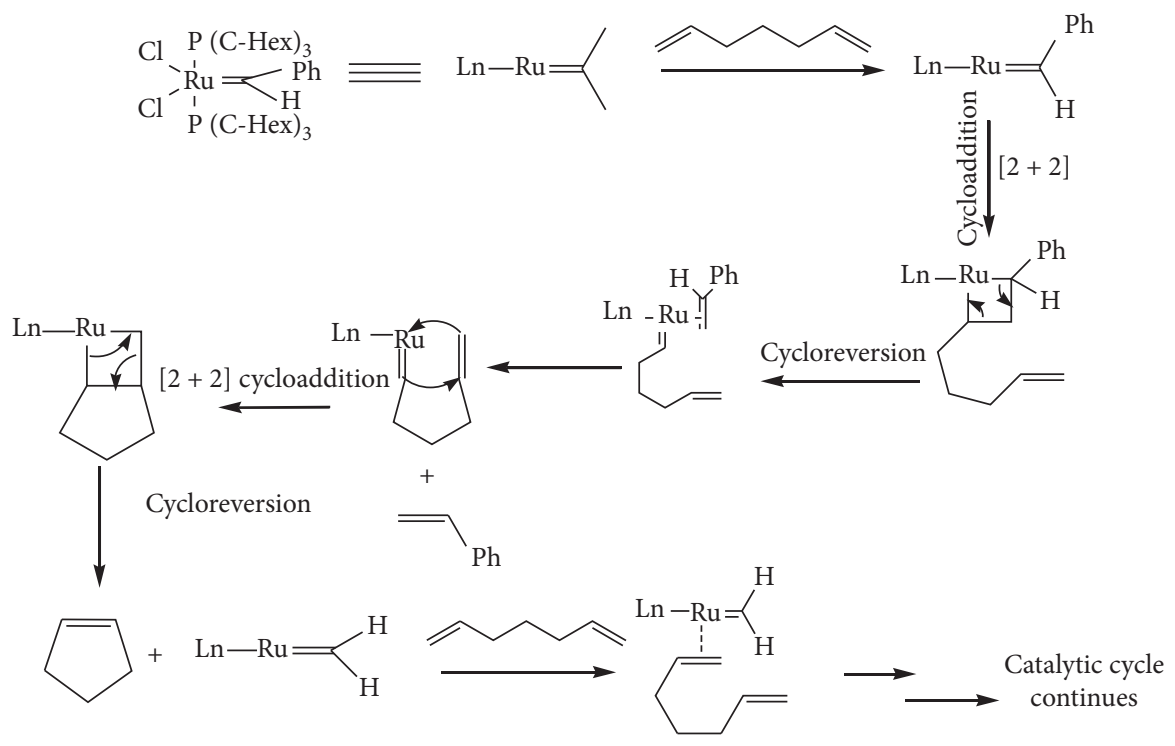

SCHeme 10: A plausible reaction mechanism for this RCM is given [10].

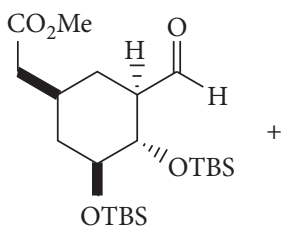<smiles>CC[C@H]1O[C@H](/C(C)=C/[C@@H](C)CS(=O)(=O)c2ccccc2)CCC1C</smiles><smiles>C=CC</smiles><smiles>C#CCCCCCC</smiles>

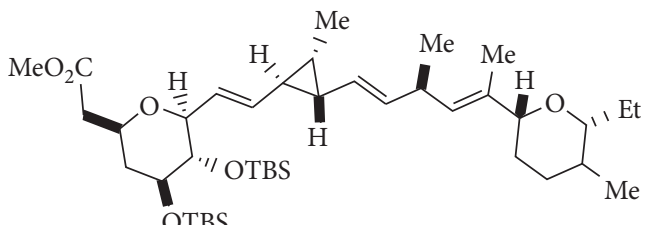

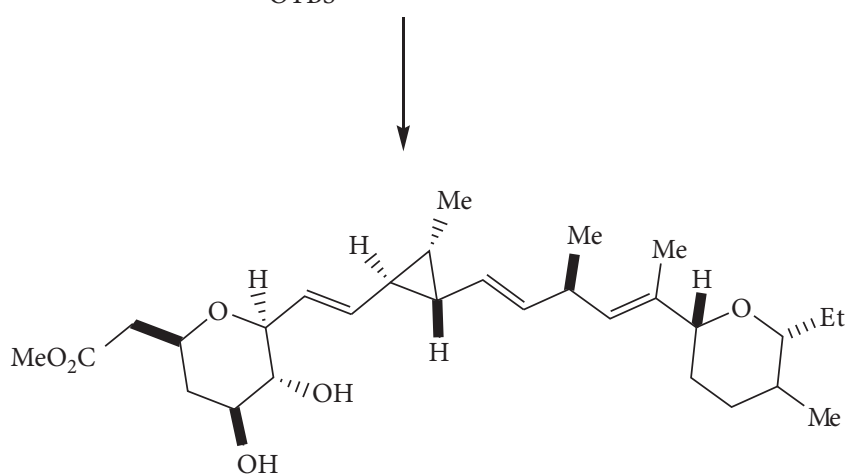

Scheme 11: Synthesis of (+)-ambruticin S via RCM reactions [60].

metallacyclobutane intermediate to form a growing polymer chain. This intermediate undergoes cycloreversion to afford a new metal alkylidene. Analogous steps are repeated during the propagation stage until polymerization ceases; that is, the monomer is completely consumed, and the reaction reaches equilibrium or the reaction is terminated $[17,67,68]$.
ROMP is commonly quenched by the addition of ethyl vinyl ether, which reacts with the metal-carbene species of a growing polymer chain end and removes the metal from the polymer. Omitting this procedure leads to degradation of the polymer product and causes metal residue contamination in the isolated material [17]. 


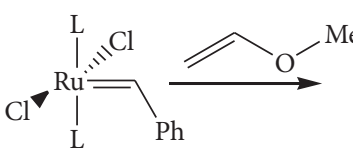

(a)

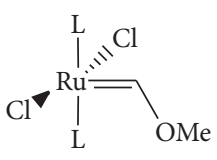

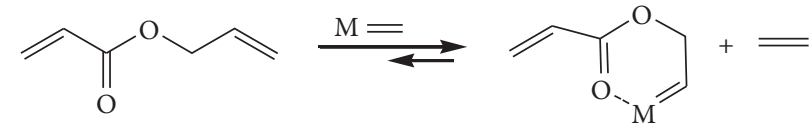

(b)

Figure 3: (a) Reaction between a general metathesis catalyst and an electron-rich olefin. (b) Possible deactivation of the catalyst via intramolecular heteroatom chelation.

$\mathrm{n}$

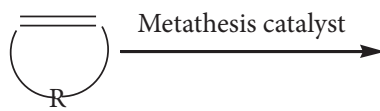

Scheme 12: ROMP general reaction.

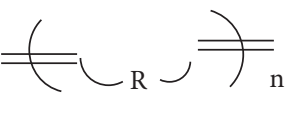

$$
\text { . }
$$




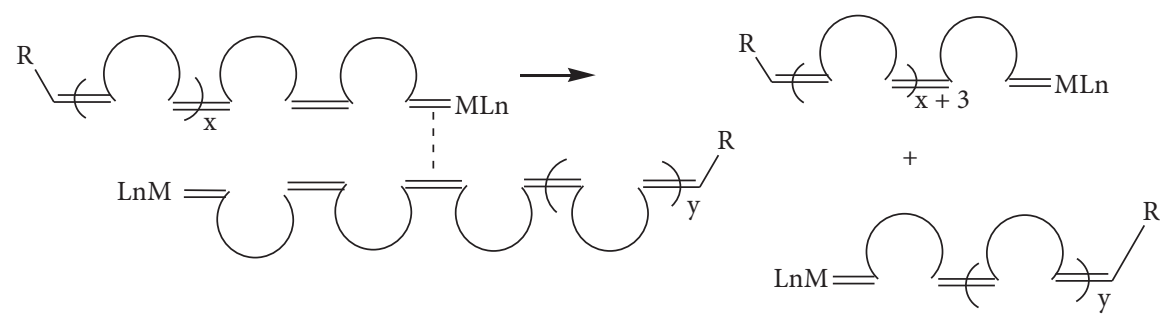

(a)

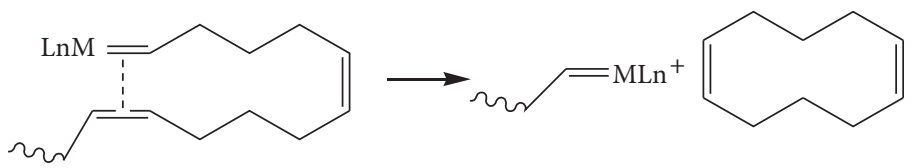

(b)

Scheme 14: Chain-transfer reactions accompanied in ROMP. (a) Intermolecular chain transfer. (b) Intramolecular chain transfer.

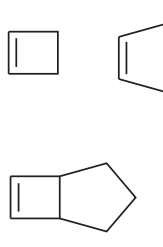<smiles>C/C=C\CC1CC2C=CC1C21CCCC1</smiles><smiles>C1=CC=CC=CC=C1</smiles><smiles>C1=CCCCCCC=C1</smiles><smiles>[R]C1C2C=CC(C2)C1[R]</smiles>

Norbornene derivative

FIgURE 4: Representative ROMP monomers.

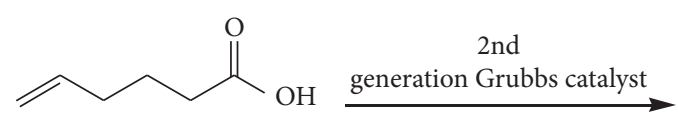

A<smiles>O=C1CC/C=C\CCCC(=O)Oc2ccc3c(c2)/C(=C2\CCc4ccc(cc42)O1)CC3</smiles>

$\mathrm{D}$
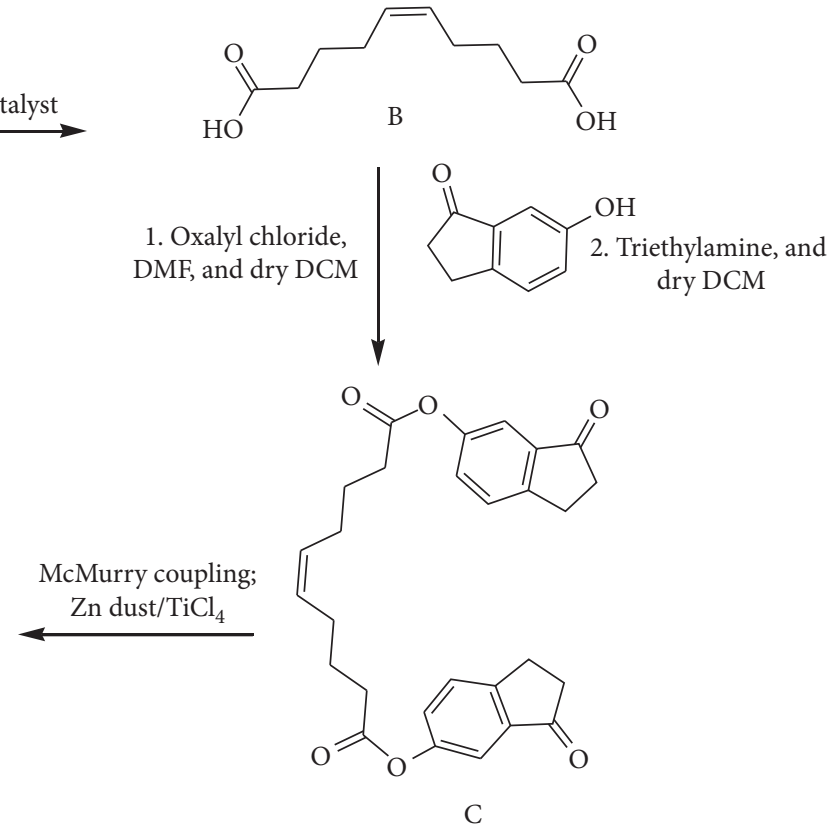

C

Scheme 15: Synthesis of macrocycle D using the main-chain stiff-stilbene via ROMP and other reaction sequences.

Acyclic Diene Metathesis Polymerization, ADMET. The ADMET polymerization is a step condensation polymerization reaction of diene monomers driven by the removal of the condensate, typically ethylene, to obtain high polymer and since the polymerization involves an equilibrium process, the polymerization is reversible $[5,78-80]$. 


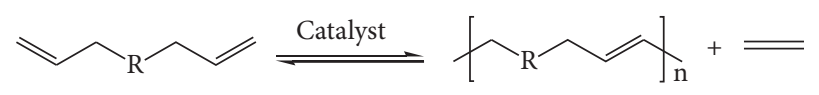

SCHEME 16: ADMET polymerization: general reaction.

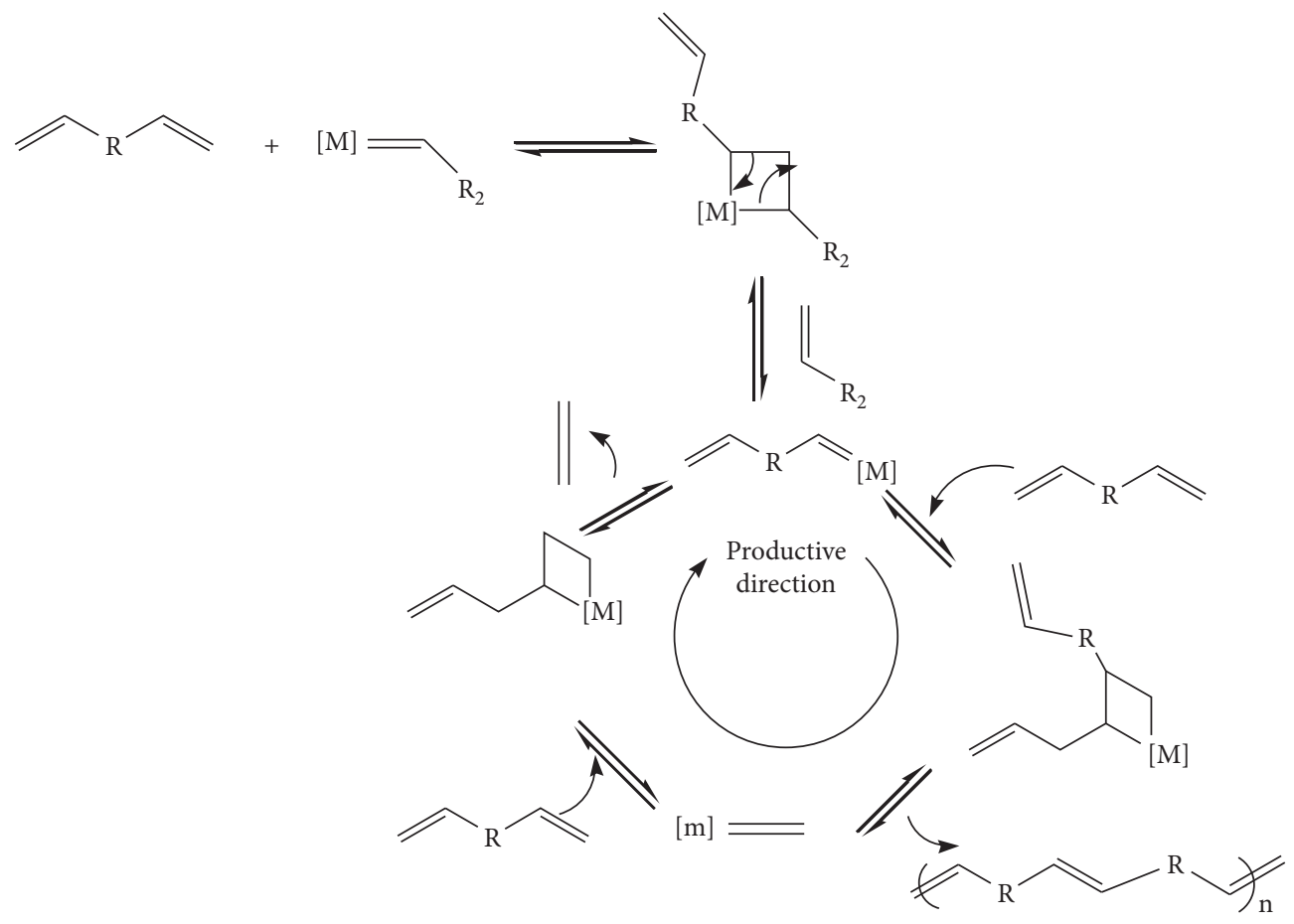

SCHEMe 17: Reaction mechanism of ADMET polymerization.

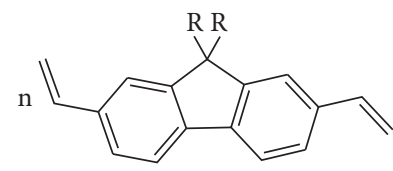

$\mathrm{R}=\mathrm{n}$-octyl, 2'-ethylhexyl, n-hexyl

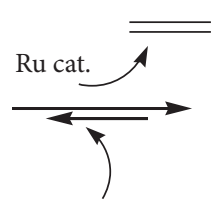

$\overline{\underline{ }}$

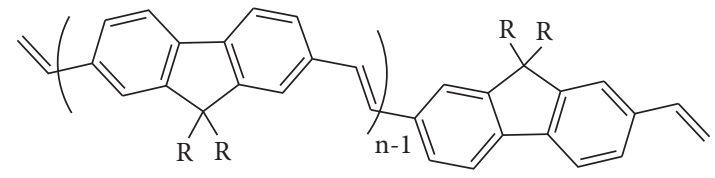

PFV

$$
+(\mathrm{n}-1)=
$$

Scheme 18: Synthesis of trans-poly(9, 9-di-n-octyl-fluorene-2,7-vinylene) (PFV) from 2,7-divinyl-9,9-di-n-octyl-fluorene using Schrock type molybdenum alkylidene [86].

The ADMET and ROMP mechanisms both proceed through the equilibrium polymerization of olefins via metathesis. However, ADMET differs by involving the polymerization of acyclic dienes, with its equilibrium shifted to the polymer by the removal of a small alkene (entropically) (Scheme 16). ROMP is typically shifted to high polymers via a favorable enthalpy change via ring strain release. The major mechanistic difference is that ROMP follows a chain-growth process where the active alkylidene acts as a catalytic initiator. In contrast, $\mathrm{ADMET}$ follows a step condensation mechanism and the alkylidene behaves as a true catalyst $[81,82]$.

The ADMET polymerization proceeds via a metalcarbene mechanism (Scheme 17), in which the metal methylidene is the true catalyst formed during the polymerization cycle (the following scheme). This is the same metal-carbene mechanism operating in cross metathesis. Coordination of the metallic center of the catalyst with a terminal olefin from either the monomer or polymer is followed by a reversible cycloaddition to produce a metallocyclobutane intermediate. This undergoes further reversion leading to either a productive or a nonproductive pathway. Chain growth is promoted by the productive metathesis pathway resulting from cycloreversion, which will not restore the coordinating olefin and free catalyst. This pathway is characterized by the production of the condensate, commonly ethylene, which should be removed from the reaction mixture in order to drive the equilibrium towards chain growth $[83,84]$. 
ADMET polymerization has been used in synthesizing even noble conjugated organic molecules which are semiconductors applicable as different electronic devices. This reaction is preferred from the other conventional methods like Heck coupling and Horner-Wittig Emmons reactions because of structural regularity and molecular purity (free from halogens, phosphorus, etc.) of synthesized polymers. ADMET polymerization reaction is effective to produce structurally defect-free conjugated polymers (Scheme 18) [85].

ADMET polymerization is achieved when the following three main variables are kept i.e., monomer purity, catalyst stability, and the melting point of the final polymers $[64,82]$.

A functional group with catalyst poisoning potential must be separated from the metal center by two or more methylene spacers for the reaction to proceed cleanly to polymer. This monomer structure phenomenon has been reported elsewhere and has been termed the "negative neighboring group effect" [64].

\section{Conclusion}

Olefin metathesis reactions have been developed in many synthetic routes such as polymer industries, petrochemicals, pharmaceutical industries, and other manufacturing industries for the formation of $\mathrm{C}-\mathrm{C}$ bonds due to the facile reaction sequences and environmentally benign conditions. A variety of olefin metathesis reactions have emerged in different focusing areas. These olefin metathesis reactions are facilitated and applicable in large due to the development and modification of reaction catalysts as well as optimization of reaction conditions. Nowadays, even the impossible olefinic metathesis reactions are successful as a result of sophisticated research efforts by discovering different reactive and many functional group tolerance and compatible catalysts which can perform stereoselective reactions so that they are able to build targeted materials. Currently, this synthetic route is interconnected with the other reaction processes to yield valuable products.

\section{Data Availability}

All data are incorporated in the body of the manuscript, and since it is a review, the data are available from referenced sources.

\section{Conflicts of Interest}

The author declares no conflicts of interest.

\section{References}

[1] P. A. Byrne and D. G. Gilheany, "The modern interpretation of the Wittig reaction mechanism," Chemical Society Reviews, vol. 42, no. 16, 6670 pages, 2013.

[2] A. B. Flynn and W. W. Ogilvie, "Stereocontrolled synthesis of tetrasubstituted olefins," Chemical Reviews, vol. 107, no. 11, pp. 4698-4745, 2007.

[3] C. P. Casey, "2005 Nobel prize in chemistry. Development of the olefin metathesis method in organic synthesis," Journal of Chemical Education, vol. 83, no. 2, 192 pages, 2006.
[4] P. J. Carey, "Synthesis of novel dienes and cyclic compounds via olefin metathesis reactions catalyzed by the second generation Grubbs catalyst," University of Tennessee, Chattanooga, TN, USA, Honors Theses, 2015.

[5] F. Lefebvre, Y. Bouhoute, K. C. Szeto, N. Merle, and A. de Mallmann, Régis Gauvin and Mostafa Taoufik. Olefin Metathesis by Group VI (Mo, W) Metal Compounds, ResearchGate, Boston, MA, USA, 2018.

[6] K. J. Ivin and J. C. Mol, Olefin Metathesis and Metathesis Polymerization, Academic Press, San Diego, CA, USA, 1997.

[7] R. H. Grubbs, "Olefin metathesis," Tetrahedron, vol. 60, no. 34 , pp. 7117-7140, 2004.

[8] S. Blechert and M. Schuster, "Olefin metathesis in organic chemistry," Angewandte Chemie, vol. 36, pp. 2036-2056, 1997.

[9] S. Monsaert, A. Lozano Vila, R. Drozdzak, P. Van Der Voort, and F. Verpoort, "* Latent olefin metathesis catalysts," Chemical Society Reviews, vol. 38, no. 12, pp. 3360-3372, 2009.

[10] P. de Weghe, J. Eustache, and J. Cossy, "Metathesis reactions. General considerations," Current Topics in Medicinal Chemistry, vol. 5, no. 15, pp. 1461-1472, 2005.

[11] D. Astruc, "The metathesis reactions: from a historical perspective to recent developments," New Journal of Chemistry, vol. 29, no. 1, pp. 42-56, 2005.

[12] P. J.-L. Hérisson and Y. Chauvin, Die Makromolekulare Chemie, vol. 141, no. 1, pp. 161-176, 1971.

[13] J. Mol, "Industrial applications of olefin metathesis," Journal of Molecular Catalysis A: Chemical, vol. 213, no. 1, pp. 39-45, 2004.

[14] M. Mazur, M. Mrozowicz, W. Buchowicz et al., "Formylation of a metathesis-derived ansa[4]-ferrocene: a simple route to anticancer Organometalics," Dalton Transactions, vol. 49, 2020.

[15] J. P. Morgan, C. Morrill, and R. H. Grubbs, "Selective ring opening cross metathesis of cyclooctadiene and trisubstituted cycloolefins," Organic Letters, vol. 4, no. 1, pp. 67-70, 2002.

[16] B. Huang, Y. Shen, Z. Mao, Y. Liu, and S. Cui, "Metathesis reaction of diazo compounds andpara-quinone methides for $\mathrm{C}-\mathrm{C}$ double bond formation: synthesis of tetrasubstituted alkenes and quinolinones," Organic Letters, vol. 18, pp. 4888-4891, 2016.

[17] S. Sutthasupa, M. Shiotsuki, and F. Sanda, "Recent advances in ring-opening metathesis polymerization, and application to synthesis of functional materials," Polymer Journal, vol. 42, no. 12, pp. 905-915, 2010.

[18] S. J. Connon and S. Blechert, "Recent developments in olefin cross-metathesis," Angewandte Chemie International Edition, vol. 42, no. 17, pp. 1900-1923, 2003.

[19] A. Deiters and S. F. Martin, "Synthesis of oxygen- and nitrogen-containing heterocycles by ring-closing metathesis," Chemical Reviews, vol. 104, no. 5, pp. 2199-2238, 2004.

[20] M. D. McReynolds, J. M. Dougherty, and P. R. Hanson, "Synthesis of phosphorus and sulfur heterocycles via ringclosing olefin metathesis $\dagger$," Chemical Reviews, vol. 104, no. 5, pp. 2239-2258, 2004.

[21] T. W. Baughman and K. B. Wagener, "Recent advances in ADMET polymerization," Metathesis Polymerization, vol. 176, pp. 1-42, 2005.

[22] E. Lehman and K. B. Wagener, Handbook of Metathesis, R. H. Grubbs, Ed., vol. 3, pp. 283-353, Wiley VCH, Weinheim, Germany, 2003.

[23] S. E. Lehman and K. B. Wagener, Late Transition Metal Polymerization Catalysis, B. Rieger, L. S. Baugh, S. Kacker, and S. Striegler, Eds., pp. 193-229, Wiley VCH, Weinheim, Germany, 2003. 
[24] R. Roy and S. K. Das, "Recent applications of olefin metathesis and related reactions in carbohydrate chemistry," Chemical Communications, vol. 7, no. 7, pp. 519-529, 2000.

[25] S. E. Lehman and K. B. Wagener, "Comparison of the kinetics of acyclic diene metathesis promoted by Grubbs ruthenium olefin metathesis catalysts," Macromolecules, vol. 35, no. 1, pp. 48-53, 2002.

[26] M. R. Buchmeiser, "Homogeneous metathesis polymerization by well-defined group VI and group VIII transition-metal alkylidenes: fundamentals and applications in the preparation of advanced materials," Chemical Reviews, vol. 100, no. 4, pp. 1565-1604, 2000.

[27] U. Frenzel and O. Nuyken, "Ruthenium-based metathesis initiators: development and use in ring-opening metathesis polymerization," Journal of Polymer Science Part A: Polymer Chemistry, vol. 40, no. 17, pp. 2895-2916, 2002.

[28] R. R. Schrock, "Living ring-opening metathesis polymerization catalyzed by well-characterized transition-metal alkylidene complexes," Accounts of Chemical Research, vol. 23, no. 5, pp. 158-165, 1990.

[29] R. R. Schrock and A. H. Hoveyda, "Molybdenum and tungsten imido alkylidene complexes as efficient olefin-metathesis catalysts," Angewandte Chemie International Edition, vol. 42, no. 38, pp. 4592-4633, 2003.

[30] I. A. Gorodetskaya, T.-L. Choi, and R. H. Grubbs, "Hyperbranched macromolecules via olefin metathesis," Journal of the American Chemical Society, vol. 129, no. 42, pp. 12672-12673, 2007.

[31] E. N. Guidry, J. Li, J. F. Stoddart, and R. H. Grubbs, "Bifunctional [c2]Daisy-Chains and their incorporation into mechanically interlocked polymers," Journal of the American Chemical Society, vol. 129, no. 29, pp. 8944-8945, 2007.

[32] J. B. Matson and R. H. Grubbs, "Synthesis of fluorine-18 functionalized nanoparticles for use as in vivo molecular imaging agents," Journal of the American Chemical Society, vol. 130, no. 21, pp. 6731-6733, 2008.

[33] D. Butilkov, A. Frenklah, I. Rozenberg, S. Kozuch, and N. G. Lemcoff, "Highly selective olefin metathesis with CAAC-containing ruthenium benzylidenes," ACS Catalysis, vol. 7, no. 11, pp. 7634-7637, 2017.

[34] T. M. Trnka and R. H. Grubbs, "The development of L2X2RuCHR olefin metathesis catalysts: an organometallic success story," Accounts of Chemical Research, vol. 34, no. 1, pp. 18-29, 2001.

[35] A. Fürstner, "Olefin metathesis and beyond," Angewandte Chemie, vol. 39, no. 17, pp. 3012-3043, 2000.

[36] L. Delaude and A. F. Noels, "Metathesis," Kirk-Othmer Encyclopedia of Chemical Technology, Wiley VCH, Weinheim, Germany, 2005.

[37] Y. İmamoğlu, B. Zümreoğlu-Karan, and J. Allan, "Olefin metathesis and polymerization catalysts synthesis, mechanism and utilization," ASIC, vol. 1, 326 pages, 1989.

[38] E. K. Pefkianakis and G. C. Vougioukalakis, "Purification of olefin metathesis reaction products via straightforward and low-cost protocols," Organic Chemistry: Current Research, vol. 2, no. 1, 2013.

[39] A. K. Chatterjee, T.-L. Choi, D. P. Sanders, and R. H. Grubbs, "A general model for selectivity in olefin cross metathesis," Journal of the American Chemical Society, vol. 125, no. 37, pp. 11360-11370, 2003.

[40] F. Sinclair, M. Alkattan, J. Prunet, and M. P. Shaver, "Olefin cross metathesis and ring-closing metathesis in polymer chemistry," Polymer Chemistry, vol. 8, no. 22, pp. 3385-3398, 2017.
[41] C. Arpin, "Cross-metathesis of electron-deficient polyenes and studies toward the total synthesis of arenolide," Chemistry \& Biochemistry Graduate Theses \& Dissertations, University of Colorado, Boulder, CO, USA, 2011.

[42] A. K. Chatterjee, D. P. Sanders, and R. H. Grubbs, "Synthesis of symmetrical trisubstituted olefins by cross metathesis," Organic Letters, vol. 4, no. 11, pp. 1939-1942, 2002.

[43] M. Lera and C. J. Hayes, "An olefin cross-metathesis approach to vinylphosphonate-linked nucleic acids," Organic Letters, vol. 3, no. 17, pp. 2765-2768, 2001.

[44] R. Stragies, U. Voigtmann, and S. Blechert, "Improved yneene-cross metathesis utilizing a dihydroimidazole carbene ruthenium complex," Tetrahedron Letters, vol. 41, no. 29, pp. 5465-5468, 2000.

[45] R. Ettari and N. Micale, "Chloro-substituted Hoveyda-Grubbs ruthenium carbene: investigation of electronic effects," Journal of Organometallic Chemistry, vol. 692, no. 16, pp. 3574-3576, 2007.

[46] A. Michrowska, R. Bujok, S. Harutyunyan, V. Sashuk, G. Dolgonos, and K. Grela, "Nitro-substituted Hoveyda-Grubbs ruthenium carbenes: enhancement of catalyst activity through electronic activation," Journal of the American Chemical Society, vol. 126, no. 30, pp. 9318-9325, 2004.

[47] T.-L. Choi, A. K. Chatterjee, and R. H. Grubbs, "Synthesis of $\alpha, \beta$-unsaturated amides by olefin cross-metathesis," Angewandte Chemie International Edition, vol. 40, no. 7, pp. 1277-1279, 2001.

[48] B. H. Lipshutz, S. Ghorai, and Ž. V. Bošković, "Tandem olefin metathesis-elimination reactions. A new route to doubly unsaturated carbonyl derivatives," Tetrahedron, vol. 64, no. 29, pp. 6949-6954, 2008.

[49] B. H. Lipshutz, G. T. Aguinaldo, S. Ghorai, and K. Voigtritter, "Olefin cross-metathesis reactions at room temperature using the nonionic amphiphile "PTS": just add water," Organic Letters, vol. 10, no. 7, pp. 1325-1328, 2008.

[50] B. H. Lipshutz, S. Ghorai, and W. W. Y. Leong, "Deprotection of homoallyl (hAllyl) derivatives of phenols, alcohols, acids, and amines," The Journal of Organic Chemistry, vol. 74, no. 7, pp. 2854-2857, 2009.

[51] T. Boddaert, Y. Coquerel, and J. Rodriguez, "Microwaveassisted cross-metathesis of acrylonitrile," Comptes Rendus Chimie, vol. 12, no. 8, pp. 872-875, 2009.

[52] M. Rivard and S. Blechert, "Effective and inexpensive acrylonitrile cross-metathesis: utilisation of Grubbs II precatalyst in the presence of copper(I) chloride," European Journal of Organic Chemistry, vol. 2003, no. 12, pp. 2225-2228, 2003.

[53] K. Voigtritter, S. Ghorai, and B. H. Lipshutz, "Rate enhanced olefin cross-metathesis reactions: the copper iodide effect," The Journal of Organic Chemistry, vol. 76, no. 11, pp. 46974702, 2011.

[54] F. Sinclair, M. Alkattan, J. Prunet, and M. P. Shaver, "Olefin cross metathesis and ring-closing metathesis in polymer chemistry," Polymer Chemistry, vol. 8, no. 22, pp. 3385-3398, 2017.

[55] M. Gatti, New catalysts and new substrates in ring-closing metathesis, Ph.D. thesis, University of Zurich, Zürich, Switzerland, 2011.

[56] K. Tomasz, F. Marek, and B. Michał, "Olefin metathesis: a versatile synthetic tool for use in preparation of Active Pharmaceutical Ingredients," Chemistry Today, vol. 32, no. 5, 2014.

[57] G. S. Sankaran and Balasubramaniam, "An overview on ring closing metathesis reaction and its applications," Journal of Agricultural Research, vol. 3, no. 10, 2018. 
[58] K. Susan, "Armstrong Ring closing diene metathesis in organic synthesis," Journal of the Chemical Society, vol. 1, pp. 371-388, 1998.

[59] J. Ramírez-Fernández, I. G. Collado, and R. HernándezGalán, "Effect of substituents on the ring-closing metathesis reaction in the synthesis of functionalized nonanolactones," Synlett, vol. 3, pp. 339-342, 2008.

[60] T. A. Kirkland, J. Colucci, L. S. Geraci et al., "Total synthesis of (+)-Ambruticin S," Journal of the American Chemical Society, vol. 123, no. 49, pp. 12432-12433, 2001.

[61] G. Subrata, G. Sarita, and S. Niladri, "Factors influencing ring closure through olefin metathesis-a perspective," Journal of Chemical Sciences, vol. 118, no. 3, pp. 223-235, 2006.

[62] K. Song, K. Kim, D. Hong et al., "Highly active ruthenium metathesis catalysts enabling ring-opening metathesis polymerization of cyclopentadiene at low temperatures," Nature Communications, vol. 10, no. 1, 3860 pages, 2019.

[63] R. Thomas, "Hoye and hongyu zhao. Some allylic substituent effects in ring-closing metathesis reactions: allylic alcohol activation," Organic Letters, vol. 1, 1999.

[64] A. Lyapkov, S. Kiselev, G. Bozhenkova, O. Kukurina, M. Yusubov, and F. Verpoort, "Ring opening metathesis polymerization," Recent Research in Polymerization, 2017.

[65] O. Nuyken and S. Pask, "Ring-opening polymerization-an introductory review," Polymers, vol. 5, no. 2, pp. 361-403, 2013.

[66] J. A. Tallarico, M. L. Randall, and M. L. Snapper, "Selectivity in ring-opening metatheses," Tetrahedron, vol. 53, no. 48, pp. 16511-16520, 1997.

[67] A. R. Hlil, J. Balogh, S. Moncho et al., "Ring opening metathesis polymerization (ROMP) of five- to eight-membered cyclic olefins: computational, thermodynamic, and experimental approach," Journal of Polymer Science Part A: Polymer Chemistry, vol. 55, no. 18, pp. 3137-3145, 2017.

[68] I. Choinopoulos, 'Grubbs' and schrock's catalysts, ring opening metathesis polymerization and molecular brushessynthesis, characterization, properties and applications," Polymers, vol. 11, no. 2, 298 pages, 2019.

[69] D. M. Lynn, S. Kanaoka, and R. H. Grubbs, "Living ringopening metathesis polymerization in aqueous media catalyzed by well-defined ruthenium carbene complexes," Journal of the American Chemical Society, vol. 118, no. 4, pp. 784-790, 1996.

[70] T.-L. Choi and R. H. Grubbs, "Controlled living ring-opening-metathesis polymerization by a fast-initiating ruthenium catalyst," Angewandte Chemie International Edition, vol. 42, no. 15, pp. 1743-1746, 2003.

[71] M. Ulman and R. H. Grubbs, "Relative reaction rates of olefin substrates with ruthenium(II) carbene metathesis initiators," Organometallics, vol. 172484 pages, 1998.

[72] C. W. Bielawski and R. H. Grubbs, "Living ring-opening metathesis polymerization," Progress in Polymer Science, vol. 32, no. 1, pp. 1-29, 2007.

[73] P. v. R. Schleyer, J. E. Williams, and K. R. Blanchard, "Evaluation of strain in hydrocarbons. The strain in adamantane and its origin," Journal of the American Chemical Society, vol. 92, no. 8, pp. 2377-2386, 1970.

[74] P. A. Patton, C. P. Lillya, and T. J. Mccarthy, "Olefin metathesis of cyclohexene," Macromolecules, vol. 19, no. 4, pp. 1266-1268, 1986.

[75] S. Akbulatov and R. Boulatov, "Experimental polymer mechanochemistry and its interpretational frameworks," ChemPhysChem, vol. 18, no. 11, pp. 1422-1450, 2017.
[76] B. P. Krishnan, L. Xue, X. Xiong, and J. Cui, "Photoinduced strain-assisted synthesis of a stiff-stilbene polymer by ringopening metathesis polymerization," Chemistry-A European Journal, vol. 26, no. 65, pp. 14828-14832, 2020.

[77] G. Xie, M. R. Martinez, M. Olszewski, S. S. Sheiko, and K. Matyjaszewski, "Molecular bottlebrushes as novel materials," Biomacromolecules, vol. 20, no. 1, pp. 27-54, 2019.

[78] P. Naresh, M. Sirisha, K. Vijay, and N. Srinath, "Recent advances of olefin metathesis and it's applications in organic synthesis," International Journal of Pharmacy and Technology, vol. 3, no. 4, pp. 1748-1767, 2011.

[79] K. B. Wagener, J. M. Boncella, and J. G. Nel, "Acyclic diene metathesis (ADMET) polymerization," Macromolecules, vol. 24, no. 10, pp. 2649-2657, 1991.

[80] D. Tindall, J. H. Pawlow, and K. B. Wagener, "Alkene metathesis in organic synthesis," Journal of Organometallic Chemistry, vol. 185-198, 1998.

[81] R. H. Grubbs, J. W. Ziller, and P. Schwab, "Synthesis and applications of $\mathrm{RuCl} 2\left(\mathrm{CHR}^{\prime}\right)(\mathrm{PR} 3) 2$ : the influence of the alkylidene moiety on metathesis activity," Journal of the American Chemical Society, vol. 118100 pages, 1996.

[82] L. Hong, L. C. da Silva, M. D. Schulz, G. Rojas, and K. B. Wagener, "A review of how to do an acyclic diene metathesis reaction," Polymer International, vol. 66, pp. 7-12, 2017.

[83] K. L. Opper and K. B. Wagener, "ADMET: metathesis polycondensation," Journal of Polymer Science Part A: Polymer Chemistry, vol. 49, no. 4, pp. 821-831, 2011.

[84] N. F. Sauty, L. C. da Silva, M. D. Schulz, C. S. Few, and K. B. Wagener, "Acyclic diene metathesis polymerization and precision polymers," Applied Petrochemical Research, vol. 4, no. 2, pp. 225-233, 2014.

[85] T. Haque and K. Nomura, "Acyclic diene metathesis (ADMET) polymerization for precise synthesis of defect-free conjugated polymers with well-defined chain ends," Catalysts, vol. 5, no. 2, pp. 500-517, 2015.

[86] K. Nomura, H. Morimoto, Y. Imanishi, Z. Ramhani, and Y. Geerts, "Synthesis of high molecular weight trans-poly $(9,9-$ di-n-octylfluorene-2,7-vinylene) by the acyclic diene metathesis polymerization using molybdenum catalysts," Journal of Polymer Science Part A Polymer Chemistry, vol. 39, pp. 2463-2470, 2001. 